\title{
Effects of Yogurt Containing Lactobacillus gasseri OLL2716 on Autonomic Nerve Activities and Physiological Functions
}

\author{
Kaiho Otomi1,2, Takuji Ymaguchi'3, Shin Watanabe', Akiko Kobayashi², \\ Hiroyuki Kobayashi ${ }^{3,4}$, Naoyuki Hashiguchi1 \\ ${ }^{1}$ Department of Emergency and Disaster Medicine, Juntendo University School of Medicine, Tokyo, Japan \\ ${ }^{2}$ Kobayashi Medical Clinic Tokyo, Tokyo, Japan \\ ${ }^{3}$ Center for Advanced Kampo Medicine and Clinical Research, Juntendo University School of Medicine, Tokyo, \\ Japan \\ ${ }^{4}$ Department of Hospital Administration, Juntendo University School of Medicine, Tokyo, Japan \\ Email: kootomi@juntendo.ac.jp
}

Received 16 February 2015; accepted 19 March 2015; published 25 March 2015

Copyright (C) 2015 by authors and Scientific Research Publishing Inc.

This work is licensed under the Creative Commons Attribution International License (CC BY).

http://creativecommons.org/licenses/by/4.0/

(c) (i)

\section{Abstract}

The purpose of this study was to investigate the effects of yogurt containing Lactobacillus gasseri OLL2716 (LG21) on autonomic nerve activities, peripheral blood flow, skin condition (skin pigmentations and moisture), saliva s-IgA and examination of quality of life (QOL). 20 healthy female volunteers (yogurt containing LG21 group: 10 people, yogurt containing Bifidobacterium (Bif) group: 10 people) were examined. The subjects ingested $100 \mathrm{~g}$ of yogurt twice daily for 4 weeks. Analysis was before and after 4 weeks dosage. By the effects for the autonomic nervous activity, parasympathetic increase was observed in the LG21 yogurt group, but was not significant increase. The LG21 yogurt was significantly increased on the peripheral blood flow. The LG21 yogurt was significantly increased on saliva s-IgA. The LG21 yogurt and Bif yogurt were significantly decrease on skin pigmentation. Also, LG21 yogurt was significantly increased on skin moisture. As a result of QOL questionnaire, incomplete evacuation, lower abdominal fullness, cold extremities and pimply or rough skin improved in LG21 yogurt and Bif yogurt after the administration period. These results suggest that the improvement effects of LG21 yogurt may be related to the activity of the parasympathetic nervous system.

\section{Keywords}

Yogurt, Lactobacillus gasseri OLL2716 (LG21), Autonomic Nerve Activities, Blood Flow, Skin Condition 


\section{Introduction}

In recent years, society has become more stressful due to changes in the social environments. We are troubled with constipation and diarrhea from the stress that it is a healthy subject, and is constant, irregular life. Therefore, various functional foods, such as blood pressure lowing food and blood glucose lowing food, have been developed and marketed. It is thought to be important to health, keeping a bowel movement well, and fixing the intestinal environment. The live microbial feeds added to improve the intestinal microbial balance are now known as probiotics [1]. Probiotics are defined as "Live microorganisms which when administered in adequate amounts confer a health benefit on the host” [2]. Lactobacili and bifidobacterium are the most commonly used probiotics while yogurt and freeze-dried cultures are popular foods containing probiotics. Probiotics have been used to manage diarrhea, enhance the immune response, enteric infection, prevent food poisoning and prevent colonic cancer.

The yogurt has been taken in as the food which is good for health and beauty from experience for a long time. Recently, there have been an increasing number of reports on the health-promoting effects of lactobacilli in yogurt. In the previous study reported that yogurt containing Lactobacillus gasseri OLL2716 (LG21) is effective against Helicobacter pylori (H. pylori), improved the pepsinogen I/II ratio in $H$. pylori positive healthy volunteers and patients with $H$. pylori infection, inhibits the formation of HCL-induced acute gastric lesions through the generation of prostaglandin E2 and healing of acetic acid-induced chronic gastric ulcer [3]-[6]. Moreover, the tablet containing Lactobacillus gasseri OLL2809 is effective on endometriosis, especially against menstrual pain and dysmenorrhea [7].

It has been experienced that persistent constipation worsens dermal characteristics and aggravates comedones as the intestinal environment deteriorates. We may expect a beauty effect if we improve constipation and intestinal environment by intake of the yogurt.

In the present study, we evaluated for the effects of yogurt containing Lactobacillus gasseri OLL2716 (LG21) on autonomic nerve activities, peripheral blood flow, skin condition (skin pigmentations and moisture), saliva s-IgA and examination of quality of life (QOL).

\section{Materials and Methods}

\subsection{Subjects}

The subjects were 20 healthy female volunteers obtained consent by a purpose of this study at the Kobayashi Medical Clinic Tokyo. The subjects were divided a randomized into two groups. In the yogurt containing LG21 group assumed 10 people (age; $31.8 \pm 1.6$ years old). For the control, yogurt containing Bifidobacterium (Bif.) group assumed 10 people (age; $27.6 \pm 0.9$ years old). All subjects provided informed consent for participation.

\subsection{Experimental Food}

The yogurt containing LG21 ( $\geq 109$ c.f.u./g) was obtained from Meiji Dairies Corporation (Tokyo, Japan). The composition of $100 \mathrm{~g}$ of experimental food is energy: $89 \mathrm{kcal}$, protein: $3.8 \mathrm{~g}$, lipids: 3.4 g, carbohydrates: 10.9 g, sodium: $49 \mathrm{mg}$, calcium: $134 \mathrm{mg}$. The yogurt containing Bifidobacterium animalis subsp. lactis (Bif) of the control used a commercial product. The subjects ingested $100 \mathrm{~g}$ of yogurt twice daily for 4 weeks.

\subsection{Measurements of Autonomic Nervous Activities}

Autonomic nervous activities were assessed using Pulse Analyzer Plus TAS9 (YKC Corporation. Tokyo). For the measuring finger tip pulse, HRV (the autonomic nerve balance) analysis program was used. Heart rate was measured by conducted on it. A frequency level from 0.04 to $0.15 \mathrm{~Hz}$ was classified as Low Frequency (LF), and a frequency level from 0.15 to $0.40 \mathrm{~Hz}$ was classified as High Frequency (HF). HF was considered as an indicator for parasympathetic nerve activity. The ratio between LF and HF (LF/HF) showed the overall balance of sympathetic and/or parasympathetic nerve, it is proportional to the degree of sympathetic nerve activity and inversely proportional to parasympathetic nerve activity.

\subsection{Measurements of Peripheral Blood Flow}

The peripheral blood flow measured the blood flow of the finger (the second, three or four fingers) of the non- 
handedness side using a full-field laser perfusion imager (Moor FLPI, Moor Instruments, Essex, UK) [8] [9]. The measurement was performed before the start of the study and 4-week after the administration. We analyzed mean blood flow of one minute and showed the increase and decrease of the blood flow after the 4-week for 100 with the blood flow before the start.

\subsection{Measurements of Salivary Components}

Saliva samples were collected using the tasteless and odorless oral swabs (Salimetrics, USA) retained in the mouth for $5 \mathrm{~min}$ in a seated position, and the collected saliva was centrifuged at $1500 \mathrm{rpm}$ for $40 \mathrm{~min}$ at $4^{\circ} \mathrm{C}$. The s-IgA levels were measured with the Salivary Secretory IgA Indirect Enzyme Immunoassay Kit (Salimetrics, USA). The data showed the increase and decrease of the s-IgA after the 4-week for 100 with before the start.

\subsection{Measurements of Skin Condition}

VISIA (CanfieldScientific, USA) was used for the evaluation of skin conditions. Inside of the box on taking images kept highly uniformed brightness with six fluorescent lamps. The front, left, and right sides of the face were photographed to evaluate skin pigmentations. Skin moisture content was measured the right cheek of a face by the SKIN DIAGNOSTIC SD27 SD27 (Courage + Khazaka Electronic GmbH, Germany). The data showed the increase and decrease of the pigmentation and moisture after the 4 week for 100 with before the start.

\subsection{Examination of Quality of Life (QOL) by Questionnaire}

The quality of life (QOL) by questionnaire was assessed Table 1 . All assessments were conducted by a researcher before the intervention and every week treatment period.

Table 1. Examination of QOL by questionnaire.

\begin{tabular}{|c|c|c|c|c|c|c|c|c|}
\hline \multicolumn{2}{|c|}{ In the Past Week } & \multirow{2}{*}{$\begin{array}{c}\begin{array}{c}\text { Not Suffered } \\
\text { at All }\end{array} \\
1\end{array}$} & \multirow{2}{*}{$\begin{array}{c}\begin{array}{c}\text { Hardly } \\
\text { Suffered }\end{array} \\
2\end{array}$} & \multirow{2}{*}{$\begin{array}{c}\text { Slightly } \\
\text { Suffered }\end{array}$} & \multirow{2}{*}{$\begin{array}{c}\text { Moderately } \\
\text { Suffered }\end{array}$} & \multirow{2}{*}{$\begin{array}{c}\begin{array}{c}\text { Very } \\
\text { Suffered }\end{array} \\
5\end{array}$} & \multirow{2}{*}{$\begin{array}{c}\text { Extremely } \\
\text { Suffered }\end{array}$} & \multirow{2}{*}{$\begin{array}{c}\text { Insufferable } \\
7\end{array}$} \\
\hline 1) & Have you suffered from stomach pain? & & & & & & & \\
\hline 2) & Heartburn & 1 & 2 & 3 & 4 & 5 & 6 & 7 \\
\hline 3) & Acid reflux from the stomach & 1 & 2 & 3 & 4 & 5 & 6 & 7 \\
\hline 4) & Stomach pain on an empty stomach & 1 & 2 & 3 & 4 & 5 & 6 & 7 \\
\hline 5) & Nausea & 1 & 2 & 3 & 4 & 5 & 6 & 7 \\
\hline 6$)$ & Stomach making noise & 1 & 2 & 3 & 4 & 5 & 6 & 7 \\
\hline 7) & Fullness in the stomach & 1 & 2 & 3 & 4 & 5 & 6 & 7 \\
\hline 8) & Burp & 1 & 2 & 3 & 4 & 5 & 6 & 7 \\
\hline 9) & Fart & 1 & 2 & 3 & 4 & 5 & 6 & 7 \\
\hline 10) & Constipation & 1 & 2 & 3 & 4 & 5 & 6 & 7 \\
\hline 11) & Diarrhea & 1 & 2 & 3 & 4 & 5 & 6 & 7 \\
\hline 12) & Loose stool & 1 & 2 & 3 & 4 & 5 & 6 & 7 \\
\hline 13) & Hard stool (having difficulty to stool) & 1 & 2 & 3 & 4 & 5 & 6 & 7 \\
\hline 14) & An urgency to defecate & 1 & 2 & 3 & 4 & 5 & 6 & 7 \\
\hline 15) & Incomplete evacuation & 1 & 2 & 3 & 4 & 5 & 6 & 7 \\
\hline 16) & Lower abdominal fullness & 1 & 2 & 3 & 4 & 5 & 6 & 7 \\
\hline 17) & Impatience & 1 & 2 & 3 & 4 & 5 & 6 & 7 \\
\hline 18) & Light sleep & 1 & 2 & 3 & 4 & 5 & 6 & 7 \\
\hline 19) & Cold extremities & 1 & 2 & 3 & 4 & 5 & 6 & 7 \\
\hline 20) & Pimply or rough skin & 1 & 2 & 3 & 4 & 5 & 6 & 7 \\
\hline
\end{tabular}




\subsection{Statistical Analysis}

All the data are presented as mean \pm SEM. The statistical analysis was evaluated by T-test before the start of the study and 4 week after the administration. Statistical comparison of experimental Food was analyzed using the Wilcoxon test. The quality of life (QOL) by questionnaire was evaluated test in consideration of a variable effect.

$\mathrm{P}$ values of $<0.05$ were defined as statistically significant.

\section{Results}

\subsection{The Autonomic Nervous Activities}

The result of the autonomic nervous activities was shown in Figure 1. The LG21 yogurt group slightly increased the parasympathetic nerve activity (Figure 1(a)). The LG21 yogurt group was decreased the LF/HF ratio. The Bif yogurt group increased the LF/HF ratio (Figure 1(b)). Both in the LG21 yogurt group and Bif yogurt group, significant changes were not observed on the change of autonomic nervous activities after 4 week.

\subsection{The Peripheral Blood Flow}

The result of the peripheral blood flow was shown in Figure 2. The LG21 yogurt group significantly increased

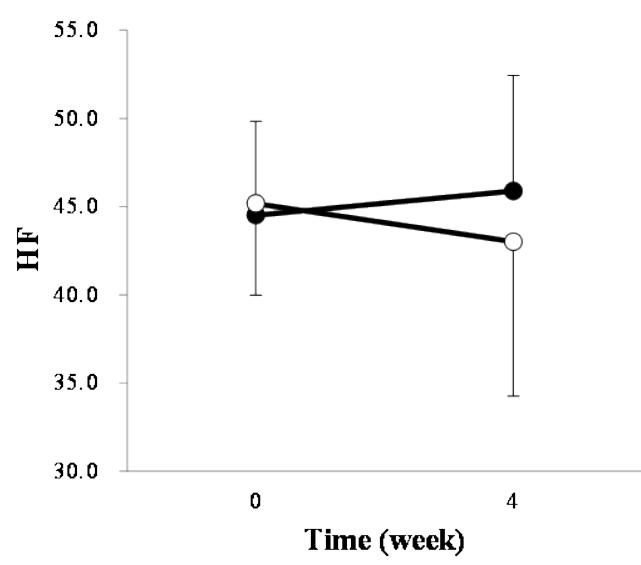

(a)

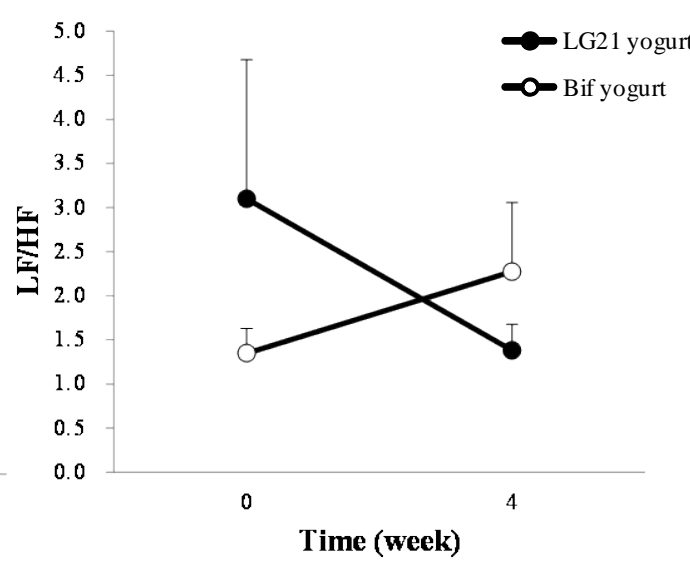

(b)

Figure 1. Effects of the yogurt on the autonomic nervous activities. (a) HF; (b) LF/HF ratio (Mean \pm SE. $n=10)$.

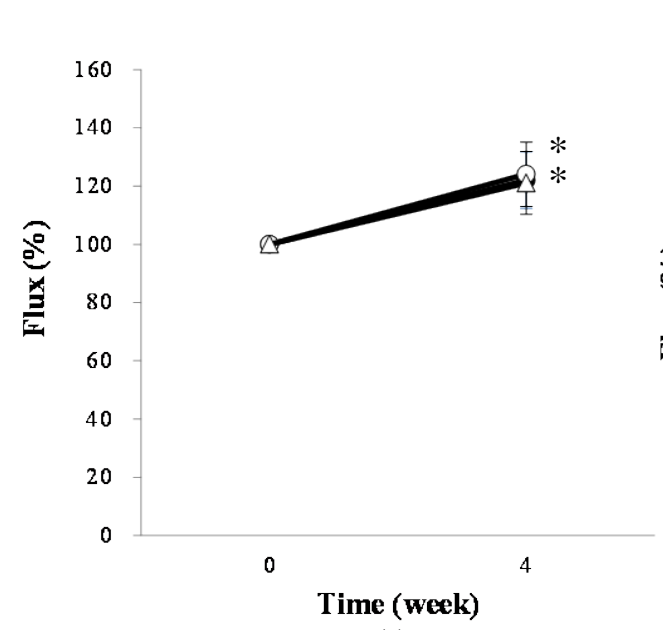

(a)

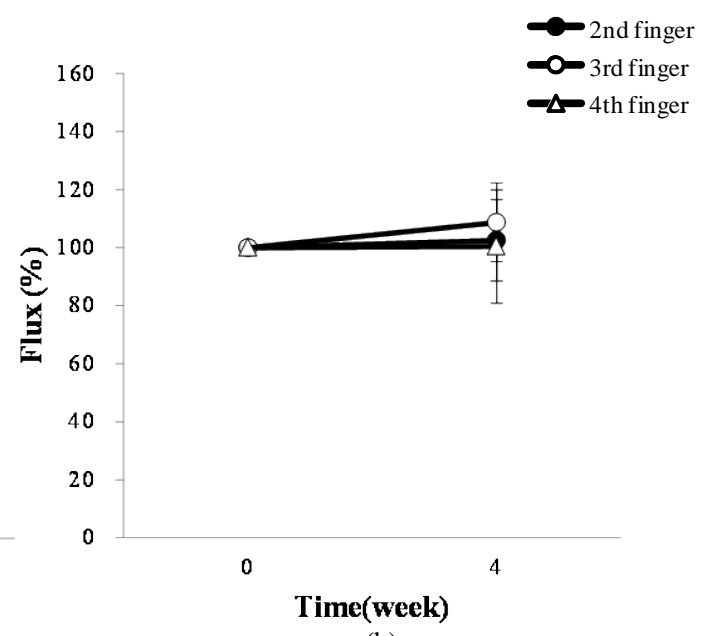

(b)

Figure 2. Effects of the yogurt on the he peripheral blood flow. (a) LG21 yogurt; (b) Bif yogurt (Mean \pm SE. $\mathrm{n}=10),{ }^{*} \mathrm{p}<0.05$ vs 0 . 
the peripheral blood flow of second and third finger at $115.6 \%, 119.3 \%$, respectively. The Bif yogurt group did not change in the peripheral blood flow.

\subsection{The Salivary Components}

The result of the saliva s-IgA level was shown in Figure 3. The LG21 yogurt group and the Bif yogurt group significantly increased the s-IgA levels. A significant difference was observed between the LG21 yogurt group and the Bif yogurt group.

\subsection{The Skin Condition}

The result of the skin pigmentation was shown in Figure 4. The LG21 yogurt and Bif yogurt significantly decreased on skin pigmentation at $85.7 \%, 80.5 \%$, respectively. The result of the skin moisture was shown in Figure 5. The LG21 yogurt significantly increased on skin moisture at $114.1 \%$. The Bif yogurt group did not change in the skin moisture. A significant difference was observed between the LG21 yogurt group and the Bif yogurt group.

\subsection{The Examination of Quality of Life (QOL)}

The result of QOL questionnaire was shown in Table 2, Table 3. A significant difference was observed between

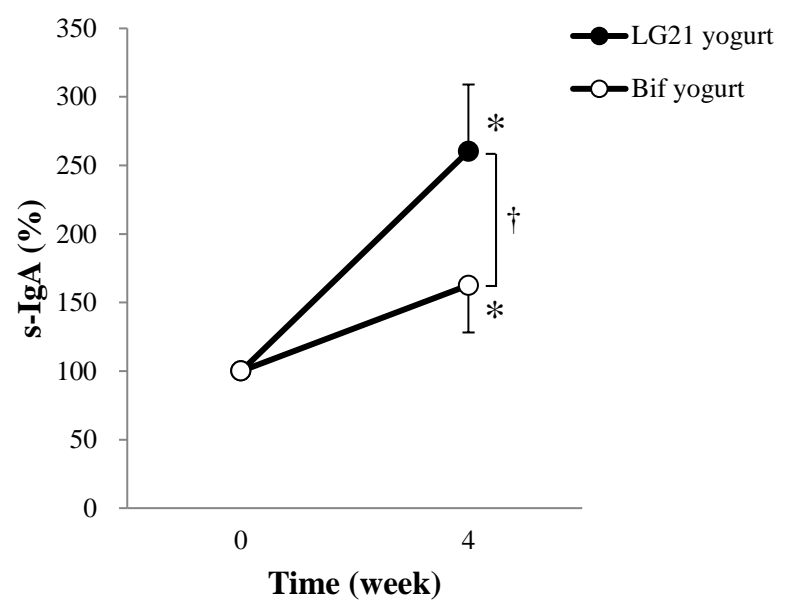

Figure 3. Effects of the yogurt on the saliva s-IgA leve. (Mean \pm SE. $\mathrm{n}=10) . *: \mathrm{p}<0.05$ vs $0, \uparrow: \mathrm{p}<0.05$ LG21 yogurt vs, Bif yogurt.

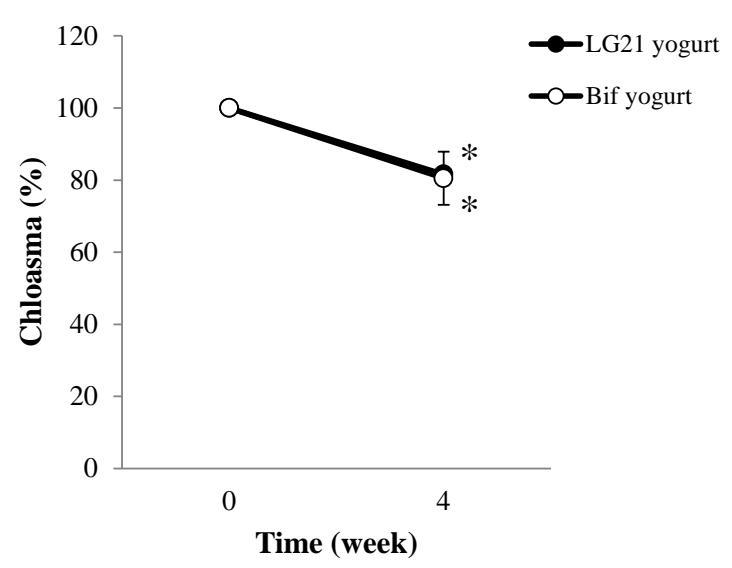

Figure 4. Effects of yogurt on skin pigmentation. (Mean \pm SE. $\mathrm{n}=10),{ }^{*} \mathrm{p}<0.05$ vs 0 . 


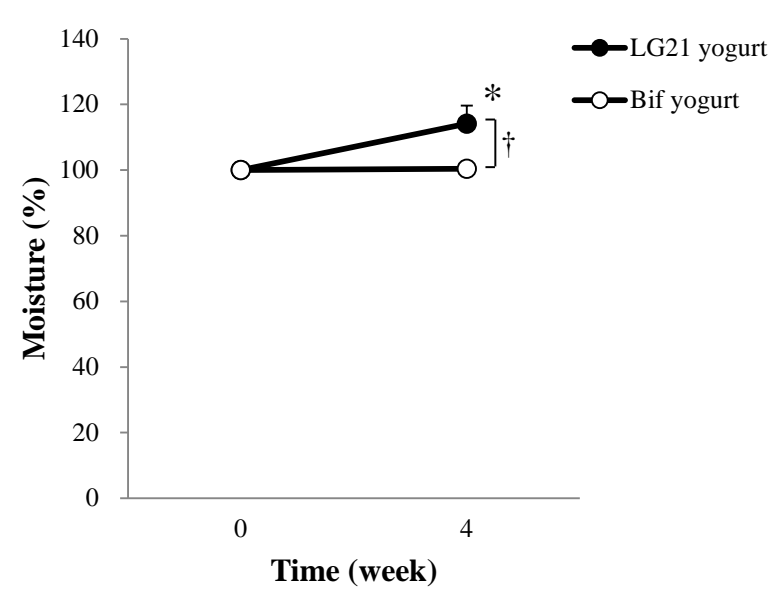

Figure 5. Effects of yogurt on skin moisture. (Mean \pm SE. $\mathrm{n}=$ 10). *: $\mathrm{p}<0.05$ vs $0, \uparrow: \mathrm{p}<0.05$ LG21 yogurt vs, Bif yogurt.

Table 2. Effects of QOL by questionnaire on an intake period becomes long.

\begin{tabular}{|c|c|c|c|c|}
\hline & & Week course ( $\mathrm{P}$ value) & Standard & $P$ value \\
\hline 1) & Have you suffered from stomach pain? & 0.286 & & \\
\hline 2) & Heartburn & 0.079 & & \\
\hline 3) & Acid reflux from the stomach & 0.153 & & \\
\hline 4) & Stomach pain on an empty stomach & 0.761 & & \\
\hline 5) & Nausea & 0.587 & & \\
\hline \multirow{4}{*}{ 6) } & \multirow{3}{*}{ Stomach making noise } & \multirow{3}{*}{0.049} & Before vs.2w & 0.099 \\
\hline & & & Before vs.3w & 0.062 \\
\hline & & & Before vs.4w & 0.099 \\
\hline & \multirow{3}{*}{ Fullness in the stomach } & \multirow{3}{*}{0.024} & Before vs.2w & 0.093 \\
\hline \multirow[t]{2}{*}{ 7) } & & & Before vs.3w & 0.076 \\
\hline & & & Before vs.4w & 0.020 \\
\hline 8) & Burp & 0.189 & & \\
\hline 9) & Fart & 0.600 & & \\
\hline 10) & Constipation & 0.999 & & \\
\hline 11) & Diarrhea & 0.276 & & \\
\hline 12) & Loose stool & 0.688 & & \\
\hline 13) & Hard stool (having difficulty to stool) & 0.150 & & \\
\hline 14) & An urgency to defecate & 0.229 & & \\
\hline 15) & Incomplete evacuation & 0.019 & Before vs.2w & 0.021 \\
\hline 16) & Lower abdominal fullness & 0.043 & Before vs.2w & 0.039 \\
\hline 17) & Impatience & 0.214 & & \\
\hline 18) & Light sleep & 0.385 & & \\
\hline \multirow{2}{*}{ 19) } & \multirow{2}{*}{ Cold extremities } & \multirow{2}{*}{0.018} & Before vs.2w & 0.038 \\
\hline & & & Before vs.3w & 0.023 \\
\hline \multirow{2}{*}{ 20) } & \multirow{2}{*}{ Pimply or rough skin } & \multirow{2}{*}{0.031} & Before vs.3w & 0.032 \\
\hline & & & Before vs.4w & 0.046 \\
\hline
\end{tabular}


Table 3. Effects of QOL by questionnaire between LG21 yogurt group and the Bif yogurt group.

\begin{tabular}{|c|c|c|}
\hline & & $\begin{array}{c}\text { Pre vs. 4W (P value) } \\
\text { Bif. vs. LG21 }\end{array}$ \\
\hline 1) & Have you suffered from stomach pain? & 0.579 \\
\hline 2) & Heartburn & 0.188 \\
\hline 3) & Acid reflux from the stomach & 0.034 \\
\hline 4) & Stomach pain on an empty stomach & 0.023 \\
\hline 5) & Nausea & 0.027 \\
\hline 6) & Stomach making noise & 0.896 \\
\hline 7) & Fullness in the stomach & 0.839 \\
\hline 8) & Burp & 0.793 \\
\hline 9) & Fart & 0.154 \\
\hline 10) & Constipation & 0.532 \\
\hline 11) & Diarrhea & 0.510 \\
\hline 12) & Loose stool & 0.086 \\
\hline 13) & Hard stool (having difficulty to stool) & 0.745 \\
\hline 14) & An urgency to defecate & 0.995 \\
\hline 15) & Incomplete evacuation & 0.602 \\
\hline 16) & Lower abdominal fullness & 0.948 \\
\hline 17) & Impatience & 0.780 \\
\hline 18) & Light sleep & 0.443 \\
\hline 19) & Cold extremities & 0.422 \\
\hline 20) & Pimply or rough skin & 0.231 \\
\hline
\end{tabular}

the LG21 yogurt group and the Bif yogurt group. As a result of QOL questionnaire, acid reflux from the stomach, stomach pain on an empty stomach and nausea improved in LG21 yogurt group. In the generally, an index of various QOL was improved if an intake period becomes long. As a result of QOL questionnaire, incomplete evacuation, lower abdominal fullness, cold extremities and pimply or rough skin improved in LG21 yogurt group and Bif yogurt group after the administration period.

\section{Discussion}

The yogurt has been taken in as the food which was good for health and beauty from experience for a long time. The beneficial effects of yogurt have been extensively reviewed. As a result of QOL questionnaire, incomplete evacuation, lower abdominal fullness, cold extremities and pimply or rough skin significantly improved in yogurt after the administration period.

In the present study, LG21 yogurt slightly increased the parasympathetic nerve activity, decreased LF/HF ratio, although a significant difference was not observed. Moreover, LG21 yogurt increased peripheral blood flow. It is generally known that autonomic nerves comprising syrnpathetic and parasympathetic nerves regulate various body functions such as blood pressure, body temperature, glucose metabolism, energy metabolism and digestion. The peripheral blood flow reflects the sole regulation of blood vessels by the sympathetic nerves, sympathetic nervous activation results in peripheral vasoconstriction and consequent reduction in blood flow. By contrast, inhibition of sympathetic nerves results in peripheral vasodilation and resultant increased blood flow. In the previous studies reported that the probiotic strain Lactobacillus johnsonii strain La1 suppressed sympathetic nerves innervating the adrenal gland and kidney of urethane-anesthetized rats, lowering the blood glucose and blood pressure levels, and facilitated the gastric parasympathetic nerve elevating appetite and body weight [10] [11]. After ingesting on the LG21 yogurt, subjects had increased peripheral blood flow, which is suggestive 
of the inhibition of sympathetic activity by the parasympathetic activation. Feeling of cold or a chilly sensation is called "Hie-sho" in Japanese, meaning cold syndrome. The factors responsible for chilly sensation are assumed to be poor peripheral blood flow and impaired metabolism. Therefore, the activity of sympathetic neurons may be important for chilly sensation [12]. Our result showed that LG21 yogurt fixed the autonomic nerve balance. These findings suggest that LG21 yogurt may be useful for the treatment of chilly sensation.

The LG21 yogurt and Bif yogurt significantly increased in the saliva s-IgA levels. IgA is the main immunoglobulin involved in mucosal defense. The increase in IgA has been attributed to the anti-infection properties of probiotics in diarrhea [13]. Recent findings have shown that secretion of IgA is critical in the regulation of the composition of the microbial community in the gut [14] [15]. In the present study, it is shown that yogurt may exert antitumour activity by a decrease in the inflammatory immune response mediated by IgA increase [16].

The LG21 yogurt and Bif yogurt significantly decreased on skin pigmentation. In addition, the LG21 yogurt significantly increased on skin moisture. It has been reported that the yogurt improved on the skin moisture [17]. For many years, it has been reported that persistent constipation worsens dermal characteristics and aggravates comedones as the intestinal environment deteriorates. Ara et al. reported that the analysis of the correlation between the defecation frequency and dermal characteristics, especially comedones, a significant increase in the number of comedones was noticed in the groups with a defecation frequency of 5 or 6 times and of 4 or fewer times per week, compared with the 7 or more-times group, confirming a correlation of worsening dermal characteristics with a greater tendency to constipation.

The improvement effect of yogurt on skin function may be related to the improvement of constipation [18].

Irritable bowel syndrome symptom includes abnormal motility, visceral hypersensitivity, inflammation, autonomic activity, and modulation of central nervous system. Brain-gut interactions play a prominent role in the modulation of gut function in health and disease. In the present study, the improvement effect of LG21 yogurt on skin function may be related to the improvement of constipation by autonomic activity and modulation of central nervous system. Moreover, the results that improvement was observed by a QOL questionnaire were confirmed in the objective evaluation.

In this study, we evaluated for the effects of the LG21 yogurt on autonomic nerve activities, peripheral blood flow, skin condition (skin pigmentations and moisture), saliva s-IgA and examination of QOL.

\section{Conclusion}

In conclusion, these results suggest that the improvement effects of LG21 yogurt may be related to the activity of the parasympathetic nervous system.

\section{References}

[1] Fuller, R. (1989) Probiotics in Man and Animals. Journal of Applied Bacteriology, 66, 365-378. http://dx.doi.org/10.1111/j.1365-2672.1989.tb05105.x

[2] Probiotics in Food. Health and Nutritional Properties and Guidelines for Evaluation._ ftp.fao.org/docrep/fao/009/a0512e/a0512e00.pdf

[3] Sakamoto, I., Igarashi, M., Kimura, K., Takagi, A., Miwa, T. and Koga, Y. (2001) Suppressive Effect of Lactobacillus gasseri OLL 2716 (LG21) on Helicobacter pylori Infection in Humans. Journal of Antimicrobial Chemotherapy, 47, 709-710. http://dx.doi.org/10.1093/jac/47.5.709

[4] Fujimura, S., Kato, S., Oda, M., Miyahara, M., Ito, Y., Kimura, K., Kawamura, T., Ohnuma, M., Tateno, H. and Watanabe, A. (2006) Detection of Lactobacillus gasseri OLL2716 Strain Administered with Yogurt Drink in Gastric Mucus Layer in Humans. Letters in Applied Microbiology, 43, 578-581. http://dx.doi.org/10.1111/j.1472-765X.2006.02017.x

[5] Uchida, M., Shimizu, K. and Kurakazu, K. (2010) Yogurt Containing Lactobacillus gasseri OLL 2716 (LG21 Yogurt) Accelerated the Healing of Acetic Acid-Induced Gastric Ulcer in Rats. Bioscience, Biotechnology, and Biochemistry, 74, 1891-1894. http://dx.doi.org/10.1271/bbb.100287

[6] Deguchi, R., Nakaminami, H., Rimbara, E., Noguchi, N., Sasatsu, M., Suzuki, T., Matsushima, M., Koike, J., Igarashi, M., Ozawa, H., Fukuda, R. and Takagi, A. (2011) Effect of Pretreatment with Lactobacillus gasseri OLL2716 on FirstLine Helicobacter pylori Eradication Therapy. Journal of Gastroenterology and Hepatology, 27, 888-892. http://dx.doi.org/10.1111/j.1440-1746.2011.06985.x

[7] Itoh, H., Uchida, M., Sashihara, T., Ji, Z.S., Li, J., Tang, Q., Ni, S., Song, L. and Kaminogawa, S. (2011) Lactobacillus gasseri OLL2809 Is Effective Especially on the Menstrual Pain and Dysmenorrhea in Endometriosis Patients: Rando- 
mized, Double-Blind, Placebo-Controlled Study. Cytotechnology, 63, 153-161. http://dx.doi.org/10.1007/s10616-010-9326-5

[8] Forrester, K.R., Stewart, C., Tulip, J., Leonard, C. and Bray, R.C. (2002) Comparison of Laser Speckle and Laser Doppler Perfusion Imaging: Measurement in Human Skin and Rabbit Articular Tissue. Medical \& Biological Engineering \& Computing, 40, 687-697. http://dx.doi.org/10.1007/BF02345307

[9] Merla, A., Di Donato, L., Romani, G.L., Proietti, M. and Salsano, F. (2008) Comparison of Thermal Infrared and Laser Doppler Imaging in the Assessment of Cutaneous Tissue Perfusion in Scleroderma Patients and Healthy Controls. International Journal of Immunopathology and Pharmacology, 21, 679-686.

[10] Tanida, M., Yamano, T., Maeda, K., Okumura, N., Fukushima, Y. and Nagai, K. (2005) Effects of Intraduodenal Injection of Lactobacillus johnsonii La1 on Renal Sympathetic Nerve Activity and Blood Pressure in Urethane-Anesthetized Rats. Neuroscience Letters, 389, 109-114. http://dx.doi.org/10.1016/j.neulet.2005.07.036

[11] Yamano, T., Tanida, M., Niijima, A., Maeda, K., Okumura, N., Fukushima, Y. and Nagai, K. (2006) Effects of the Probiotic Strain Lactobacillus johnsonii Strain La1 on Autonomic Nerves and Blood Glucose in Rats. Life Sciences, 79, 1963-1967. http://dx.doi.org/10.1016/j.lfs.2006.06.038

[12] Nasu, Y., Iwashita, M., Saito, M., Fushiya, S. and Nakahata, N. (2009) Inhibitory Effects of Atractylodis Lanceae Rhizoma and Poria on Collagen- or Thromboxane $\mathrm{A}_{2}$-Induced Aggregation in Rabbit Platelets. Biological and Pharmaceutical Bulletin, 32, 856-860. http://dx.doi.org/10.1248/bpb.32.856

[13] Perdigón, G., de Moreno de LeBlanc, A., Valdez, J. and Rachid, M. (2002) Role of Yoghurt in the Prevention of Colon Cancer. European Journal of Clinical Nutrition, 56, S65-S68. http://dx.doi.org/10.1038/sj.ejcn.1601490

[14] Kawamoto, S., Tran, T.H., Maruya, M., Suzuki, K., Doi, Y., Tsutsui, Y., Kato, L.M. and Fagarasan, S. (2012) The Inhibitory Receptor PD-1 Regulates IgA Selection and Bacterial Composition in the Gut. Science, 336, 485-489. http://dx.doi.org/10.1126/science.1217718

[15] Suzuki, K., Meek, B., Doi, Y., Muramatsu, M., Chiba, T., Honjo, T. and Fagarasan, S. (2004) Aberrant Expansion of Segmented Filamentous Bacteria in IgA-Deficient Gut. Proceedings of the National Academy of Sciences of the United States of America, 101, 1981-1986. http://dx.doi.org/10.1073/pnas.0307317101

[16] Moreau, M.C., Hudault, S. and Bridonneau, C. (1990) Systemic Antibody Response to Ovalbumin in Gnotobiotic C3H/HeJ Mice with Bifidobacterium bifidum or Escherichiacoli. Microecology and Therapy, 20, 309-312.

[17] Isawa, K., Noma, T., Yamamoto, M., Kimura, K., Ito, H., Taketomo, N., Numano, K. and Kawashima, M. (2008) Verifying the Ability of Yogurt Prepared with LB81 Lactic Acid Bacteria to Improve Skin Function. Journal of Intestinal Microbiology, 22, 1-5.

[18] Sashihara, T. (2013) Effects of Lactic Acid Bacteria on Immune Regulatory Activities. Journal of Intestinal Microbiology, 27, 197-202. 